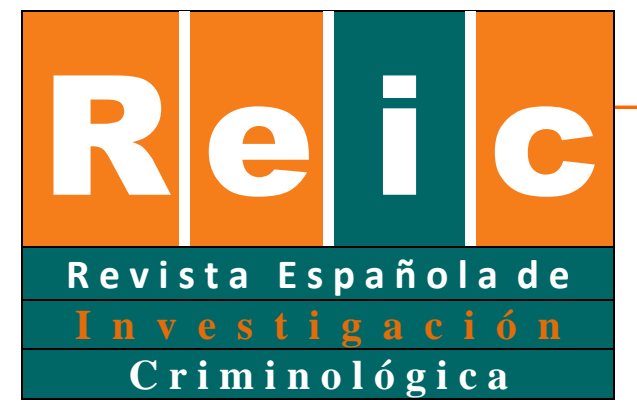

López-Riba

\title{
El análisis cuantitativo de las identificaciones y las detenciones \\ policiales en España: Datos disponibles, limitaciones e \\ implicaciones
}

\section{The quantitative analysis of police identifications and arrests in Spain: Available data, limitations and implications}

Recibido 26 febrero 2021/ Aceptado 2 julio 2021

\author{
José María López-Riba ${ }^{1}$ \\ Universidad de Girona
}

\section{RESUMEN}

El objetivo del presente artículo es poner de manifiesto la limitada capacidad que tenemos desde la Criminología para estudiar cuantitativamente el ejercicio de los poderes policiales en España a partir de los datos disponibles de fuentes oficiales. Para ello se seleccionan dos potestades policiales, la identificación y la detención, y a partir de ellas se muestran los datos disponibles, así como las posibilidades y las carencias que tienen para su uso en la investigación criminológica. Posteriormente, se exponen a modo de contraste dos ejemplos internacionales, concretamente el de las paradas policiales en Reino Unido y las detenciones en Estados Unidos. Por último, se señalan las cautelas que se han de tomar a la hora de trabajar con datos secundarios y las implicaciones que tienen la falta de datos y una política de transparencia decidida no solo para la investigación criminológica sino para un necesario monitoreo de la actividad policial en democracia.

Palabras clave: poderes policiales, datos secundarios, fuentes de datos, transparencia.

\footnotetext{
${ }^{1}$ La correspondencia debe dirigirse a: josemaria.lopez@udg.edu
} 


\begin{abstract}
The aim of this article is to highlight the limited capacity of Spanish criminology to quantitatively analyze the exercise of police powers in Spain from the available official data sources. For this purpose, two police powers are selected, ID checks and arrests, and the available data are shown, as well as the possibilities and limitations they have for their use in criminological research. Subsequently, two international examples are presented by way of contrast, that of police stops in the United Kingdom and arrests in the United States. Finally, the article concludes with the cautions to be taken when working with secondary data and the implications of the lack of data and a determined policy of transparency not only for criminological research but also for the necessary monitoring of police activity in a democracy.
\end{abstract}

Keywords: police powers, secondary data, sources of data, transparency.

\title{
1. Introducción
}

La policía tiene diferentes medios legales para cumplir con sus funciones de control de la delincuencia y mantenimiento del orden. Estos medios son lo que se denomina en el contexto anglosajón poderes policiales. El poder es un aspecto que se ejerce en muchos otros ámbitos, pero en el ámbito policial es especialmente sensible puesto que los poderes policiales se derivan de la capacidad de ejercer violencia por parte del Estado. Además, estos medios excepcionales en última instancia están respaldados por la capacidad de recurrir al uso de la fuerza.

Sobre el uso de estos medios excepcionales surgen muchas cuestiones de interés criminológico. Por ejemplo, y entre otras muchas: ¿cuánto se recurre a estos medios excepcionales? ¿qué consecuencias sociales a corto, medio y largo plazo genera el recurso actual a estos poderes? Para poder dar respuesta a estas preguntas se necesita disponer de datos concretos y accesibles, generados por instituciones oficiales (y supervisados de forma independiente).

En otros países estos temas han sido centrales en el desarrollo de la Criminología. En España debido a la falta de datos y a una cultura de poca transparencia, específicamente en temas policiales, la investigación sobre estos temas, y sobre la policía en general, es muy escasa en la comparación internacional. Pero la falta de datos y de transparencia no solo

Revista Española de Investigación Criminológica

Artículo 1, Volumen 19 (2) (2021)

https://doi.org/10.46381/reic.v19i2.499

www.criminologia.net

ISSN: $1696-9219$ 
perjudica a las investigaciones criminológicas, también perjudica al control democrático. Puesto que estos medios/poderes tensionan derechos fundamentales (como el derecho a la libertad o el derecho a la intimidad) es necesario un control intensivo y constante sobre ellos, algo que es muy difícil que ocurra sin la posibilidad de que cualquier persona pueda conocer cómo actúa la policía en su país.

Por todo ello, este trabajo pretende, en primer lugar, exponer las principales fuentes oficiales de datos sobre el ejercicio de los poderes policiales en España. En segundo lugar, se comparará la situación con otros países donde la investigación criminológica sobre estos temas está más desarrollada. En tercer lugar, se indicarán las limitaciones de utilizar este tipo de datos oficiales en general y el problema de la transparencia. En el anexo del trabajo, a modo de referencia para las fuentes utilizadas y de ayuda para futuras investigaciones, se ofrece una recopilación de las principales fuentes oficiales de datos sobre el ejercicio de los poderes policiales en España.

\section{Datos sobre poderes policiales en España}

La policía dispone de diferentes medios excepcionales en España para cumplir con las funciones $^{2}$ que le adjudica el artículo 104.1 de la Constitución Española (CE). En general estas funciones se pueden reducir a dos principales: controlar la delincuencia, mediante la prevención y la persecución de las infracciones penales, y mantener el orden ${ }^{3}$, que suele referirse a tareas de ejecución de la normativa administrativa y de control de masas (manifestaciones, protestas, espectáculos, etc.) (Guillén, 2012).

De estos poderes policiales se pueden estudiar diferentes aspectos con datos cuantitativos, como se ha señalado anteriormente. La investigación criminológica suele interesarse por los siguientes: i) la exploración de su uso (p. e., Ashby, 2020); ii) la comparación con otros contextos temporales y geográficos (ver, por ejemplo, López-Riba,

\footnotetext{
${ }^{2}$ Estas funciones se encuentran más detalladas en el artículo 11.1 de la Ley Orgánica 2/1986, de 13 de marzo, de Fuerzas y Cuerpos de Seguridad (LOFCS).

${ }^{3}$ Su significado también es diferente dependiendo del contexto. En el ámbito continental europeo suele hacer referencia a lo que señala el texto supra, en el ámbito anglosajón a la persecución del comportamiento incívico (Brodeur, 2010).
}

Revista Española de Investigación Criminológica

Artículo 1, Volumen 19 (2) (2021)

https://doi.org/10.46381/reic.v19i2.499

www.criminologia.net

ISSN: 1696-9219 
2019); iii) si el uso de estos poderes se concentra más en determinados segmentos de la población (por ejemplo, jóvenes o minorías étnicas) o en determinadas áreas geográficas (por ejemplo, aquellas con una mayor concentración de delitos) (p. e., López Riba, 2021); iv) su eficacia en el cumplimiento de su función de control de la delincuencia (por ejemplo, García España et al. 2016); v) las consecuencias sociales que tiene su uso (por ejemplo, sobre la legitimidad policial o los efectos estigmatizantes que provoca si se concentra en determinados colectivos) (Bradford, 2014).

A continuación, se analizará la disponibilidad de datos sobre el ejercicio de las identificaciones y las detenciones policiales, así como la posibilidad de estudiar las cuestiones antes apuntadas a partir de los datos disponibles.

\subsection{Identificaciones}

Las identificaciones policiales se pueden definir como la potestad que tiene un agente de policía, en determinados supuestos, para parar y requerir los datos identificativos a una persona en el espacio público o semipúblico.

Las identificaciones se encuentran reguladas principalmente en los artículos 16 y 17 de la Ley Orgánica 4/2015, de 30 de marzo, de protección de la seguridad ciudadana (LOPSC). De forma resumida, el artículo 16 habilita a un agente de policía a identificar a una persona en dos supuestos: i) cuando existan indicios de que esa persona ha podido participar en una infracción (administrativa o penal); ii) cuando el agente lo considere "razonablemente necesario" para prevenir la comisión de un delito, en atención al contexto. Por otro lado, el artículo 17 dispone que en el caso de que se trate de delitos de especial gravedad o generadores de alarma social se podrán establecer controles de identificación masivos para su prevención o para descubrir a quienes han participado en su comisión.

Son pocos los datos disponibles en abierto sobre las identificaciones policiales en España, y con destacadas limitaciones. En primer lugar, se disponen ${ }^{4}$ de los datos sobre identificaciones realizadas por el Cuerpo Nacional de Policía (CNP) y la Guardia Civil

\footnotetext{
${ }^{4} \mathrm{O}$ mejor dicho se disponían, puesto que dejaron de aparecen a partir de 2016 (con datos para 2015).
}

Revista Española de Investigación Criminológica

Artículo 1, Volumen 19 (2) (2021)

https://doi.org/10.46381/reic.v19i2.499

www.criminologia.net

ISSN: 1696-9219 
aparecidos en los Anuarios Estadísticos del Ministerio del Interior (AEMI). Estos datos aparecen agregados a nivel nacional, por CCAA y por provincias. También aparecen diferenciados por el lugar donde se llevó a cabo la identificación: en la vía pública o en dependencias policiales. En segundo lugar, a nivel autonómico, en el portal de Estadística policial de los Mossos d'Esquadra se presentan datos de identificaciones y controles de paso realizados por este cuerpo en Cataluña agregados a nivel anual. En las Memorias de la Policía Foral de Navarra (MPFN) aparecen también datos de identificaciones anuales en Navarra (diferenciándolos si se llevaron a cabo sobre personas a pie o en vehículo). Por último, algunos ayuntamientos publican datos sobre las identificaciones llevadas a cabo por sus respectivas policías locales ${ }^{5}$, pero esto está lejos de ser una práctica sistemática y extendida.

El problema más importante de estos datos disponibles en abierto es que es imposible saber cuántas identificaciones, en un periodo o territorio concreto, han realizado todos los diferentes cuerpos policiales que tienen competencia para ello. Es decir, no sabemos cuántas identificaciones policiales se llevan a cabo en España puesto que no tenemos datos para todos los cuerpos policiales. De hecho, incluso en los AEMI no se puede saber cuántas de esas identificaciones realizó el $\mathrm{CNP}^{6}$ y cuántas la Guardia Civil. La limitación más importante al respecto es la falta de datos de las policías locales en general y de cuerpos autonómicos como la Ertzaintza o el Cuerpo General de Policía Canaria.

Por lo tanto, uno de los principales objetivos básicos de los estudios sobre los poderes policiales, que es cuantificar su uso, no se puede cumplir por la falta de datos. Esto repercute en todos los otros aspectos que se pueden estudiar: comparación, eficacia, consecuencias, etc. En la tabla 1 aparece un intento por cuantificar en un año concreto el número de identificaciones llevadas a cabo en España por parte de los diferentes cuerpos policiales con

\footnotetext{
${ }^{5}$ Por ejemplo, el Ayuntamiento de Santa Coloma de Gramenet en sus anuarios estadísticos publica datos sobre las identificaciones realizadas por la policía local del municipio. Se puede ver en el último anuario publicado: https://www.gramenet.cat/fileadmin/Files/Ajuntament/anuari_estadistic/Anuari_2018_juliol2019.pdf

${ }^{6}$ Algunos Informes Anuales del Mecanismo Nacional de Prevención de la Tortura (IAMNPT) ofrecen datos de identificaciones realizadas por el CNP por provincias y CCAA y según la nacionalidad de la persona identificada. Sin embargo, el bajo número en comparación con los ofrecidos en los AEMI parece indicar que se refieren únicamente a las identificaciones realizadas en dependencias policiales. Por ejemplo: https://www.defensordelpueblo.es/wp-content/uploads/2020/06/ANEXO-1_CORTA-DURACION.pdf
}

Revista Española de Investigación Criminológica

Artículo 1, Volumen 19 (2) (2021)

https://doi.org/10.46381/reic.v19i2.499

www.criminologia.net

ISSN: 1696-9219 
las fuentes disponibles. En base a los datos disponibles para el año 2015, se calcula que hubo un mínimo ${ }^{7}$ de 7.645.242 de identificaciones policiales en España.

\section{Tabla 1}

Número de identificaciones en España en el año 2015

\begin{tabular}{ccccc}
\hline Cuerpo & $\begin{array}{c}\text { Número de } \\
\text { identificaciones }\end{array}$ & $\begin{array}{c}\text { Tasa de } \\
\text { identificaciones } \\
\text { por cada 1000 } \\
\text { habitantes }\end{array}$ & $\begin{array}{c}\text { Fuente número } \\
\text { de } \\
\text { identificaciones }\end{array}$ & Fuente población \\
\hline $\begin{array}{c}\text { CNP y Guardia } \\
\text { Civil }\end{array}$ & 6.582 .584 & 141,84 & AEMI & $\begin{array}{c}\text { INE (2020) } \\
\text { [segundo } \\
\text { semestre 2015] }\end{array}$ \\
\hline $\begin{array}{c}\text { Mossos } \\
\text { d'Esquadra }\end{array}$ & $1.003 .932^{8}$ & 135,74 & $\begin{array}{c}\text { Estadística } \\
\text { policial }\end{array}$ & $\begin{array}{c}\text { INE (2020) } \\
\text { [segundo } \\
\text { semestre 2015] }\end{array}$ \\
\hline $\begin{array}{c}\text { Policía Foral de } \\
\text { Navarra }\end{array}$ & $58.726^{9}$ & 92,3 & MPFN & $\begin{array}{c}\text { INE (2020) } \\
\text { [segundo } \\
\text { semestre 2015] }\end{array}$ \\
\hline $\begin{array}{c}\text { Ertzaintza } \\
\text { Cuerpo General }\end{array}$ & - & - & - & - \\
\hline de Policía Canaria & - & - & - & - \\
\hline \begin{tabular}{c} 
Policías locales \\
\hline
\end{tabular} & - & - & - & - \\
\hline
\end{tabular}

En la tabla 1 también se ha calculado la tasa de identificaciones según el cuerpo policial (o cuerpos policiales) en función de la población "disponible"10. En este caso, y con los datos disponibles, se puede observar cómo la tasa es mayor para los cuerpos estatales, indicando que estos cuerpos llevan a cabo conjuntamente más identificaciones que el resto. En el caso de la tasa de identificaciones de los Mossos d'Esquadra está es ligeramente menor que la anterior. Y, en el caso de la Policía Foral de Navarra, es significativamente menor a las dos anteriores.

\footnotetext{
${ }^{7}$ Teniendo en cuenta la falta de datos para policías autonómicas y locales.

${ }^{8}$ Cálculo propio a partir de sumar las identificaciones y los controles de paso (por referirse al supuesto del artículo 17 de la LOPSC también pueden considerarse identificaciones).

${ }^{9}$ Cálculo propio a partir de sumar las identificaciones a personas y vehículos.

${ }^{10}$ Para la tasa de identificaciones del CNP y la Guardia Civil se ha utilizado la población total, para la de los Mossos d'Esquadra la población de Cataluña y para la de la Policía Foral de Navarra la población de la Comunidad Foral de Navarra.
}

Revista Española de Investigación Criminológica

Artículo 1, Volumen 19 (2) (2021)

https://doi.org/10.46381/reic.v19i2.499

WWW.criminologia.net

ISSN: 1696-9219 
En cuanto a la comparación con otros contextos, difícilmente se puede realizar cuando falta la información más básica. Pero con esta limitación presente, los datos disponibles parecen indicar un mayor uso de este poder en España que en otros países (López-Riba, 2019; García Añón et al., 2013). En la tabla se comparan la tasa de identificaciones realizadas en España con otros países ${ }^{11}$ para la misma potestad o similares. En ella se puede observar que en España la tasa de identificaciones es comparativamente alta en referencia a Inglaterra y Gales y Escocia, aunque comparativamente menor a Chile ${ }^{12}$.

\section{Tabla 2}

Comparación de la tasa de identificaciones policiales

\begin{tabular}{|c|c|c|c|}
\hline País & $\begin{array}{l}\text { Tasa de identificaciones } \\
\text { por cada } 1000 \text { habitantes }\end{array}$ & $\begin{array}{c}\text { Fuente número } \\
\text { identificaciones [año] }\end{array}$ & Fuente población [año] \\
\hline España & 164,73 & $\begin{array}{c}\text { AEMI, Estadística policial } \\
\text { y MPFN [2015] }\end{array}$ & $\begin{array}{l}\text { INE (2020) [segundo } \\
\text { semestre 2015] }\end{array}$ \\
\hline Chile & 282,1 & $\begin{array}{l}\text { Duce y Lillo (2020) [abril } \\
2017 \text { - marzo 2018] }\end{array}$ & Duce y Lillo (2020) [2019] \\
\hline $\begin{array}{l}\text { Inglaterra y } \\
\text { Gales }\end{array}$ & 6,6 & $\begin{array}{c}\text { United Kingdom } \\
\text { Government (2020) [2015] }\end{array}$ & $\begin{array}{c}\text { Office for National } \\
\text { Statistics (2020) [2015] }\end{array}$ \\
\hline Escocia & 129 & $\begin{array}{c}\text { Lennon y Murray (2018) } \\
\text { [2013] }\end{array}$ & $\begin{array}{l}\text { Lennon y Murray (2018) } \\
\text { [2013] }\end{array}$ \\
\hline
\end{tabular}

Otro problema de los datos es que no aparecen las características de las personas que han sido objeto de la identificación. Por lo tanto, es difícil saber si las identificaciones se concentran en determinados colectivos o se distribuyen de forma igualitaria en todos los segmentos de la población ${ }^{13}$. Los estudios que se han hecho en España al respecto han utilizado otras fuentes (encuestas, observación o datos secundarios requeridos

\footnotetext{
${ }^{11}$ La selección de estos países se ha realizado en base a los datos disponibles a través de portales institucionales o de investigaciones realizadas.

${ }^{12}$ Es necesario tomar varias cautelas antes de sacar conclusiones. Por un lado, para España como se apuntaba anteriormente falta información. En Chile, esa tasa es comparativamente alta en referencia a años anteriores (Duce \& Lillo, 2020). Y, en el caso de Inglaterra y Gales y Escocia, pese a que son potestades similares no son idénticas.

${ }^{13}$ Algo importante pues el propio artículo 16.1 de la LOPSC establece que: "En la práctica de la identificación se respetarán estrictamente los principios de proporcionalidad, igualdad de trato y no discriminación por razón de nacimiento, nacionalidad, origen racial o étnico, sexo, religión o creencias, edad, discapacidad, orientación o identidad sexual, opinión o cualquier otra condición o circunstancia personal o social".
}

Revista Española de Investigación Criminológica

Artículo 1, Volumen 19 (2) (2021)

https://doi.org/10.46381/reic.v19i2.499

Www.criminologia.net

ISSN: 1696-9219 
específicamente a un cuerpo policial). En general, estos trabajos apuntan a un mayor uso de las identificaciones sobre hombres, jóvenes, personas extranjeras y personas que pertenecen a minorías étnicas o religiosas (por ejemplo, APDHA \& IPAZ-UGR, 2016; García Añón et al., 2013). En la tabla 3 se presenta una comparación entre la tasa de personas nacionales y extranjeras identificadas por el CNP en dependencias policiales en 2019.

\section{Tabla 3}

Tasa de identificaciones por cada 1000 habitantes del CNP en dependencias policiales por nacionalidad en el año 2019

\begin{tabular}{cc}
\hline Nacionalidad & Tasa de identificaciones \\
\hline Españoles & 3,72 \\
\hline Extranjeros & 18,05 \\
\hline
\end{tabular}

Fuente: INE (2020) e IAMNPT 2019

En esta tabla puede observarse como las identificaciones en dependencias policiales realizadas por el CNP recaen en mayor medida sobre personas extranjeras, la tasa de identificaciones para estas personas es aproximadamente 5 veces más alta que para las personas con nacionalidad española ${ }^{14}$.

La falta de datos de todos los cuerpos policiales, y la limitación en su detalle, también dificulta saber en qué lugares del territorio se llevan a cabo más o menos identificaciones. Cuando aparecen detallados por territorio, como en el caso de los AEMI, las categorías son demasiado amplias, como comunidades autónomas o provincias. En general, el tipo de estudios que busca la distribución geográfica de las identificaciones tiene en cuenta unidades

\footnotetext{
${ }^{14}$ Esto no puede justificarse en base a que a las personas extranjeras las identifican más en dependencias policiales para comprobar su situación administrativa en España, puesto que solo se puede trasladar a comisaria para identificar a alguien con el objetivo de impedir la comisión de un delito o para denunciar una infracción administrativa que ponga en riesgo la seguridad y cuando no haya sido posible la identificación por ningún medio en el mismo lugar donde ocurrió la parada policial (art. 16.2 LOPSC). Fuera de estos supuestos se podría considerar una detención ilegal (Martínez Escamilla \& Sánchez Tomás, 2019; Ríos, et al., 2015). Tampoco puede atribuirse a que las personas extranjeras delinquen más, pues no existe evidencia criminológica de ello (Fernández Molina \& Bartolomé, 2018; García Añón et al., 2013; García España, 2018).
}

Revista Española de Investigación Criminológica

Artículo 1, Volumen 19 (2) (2021)

https://doi.org/10.46381/reic.v19i2.499

Www.criminologia.net

ISSN: 1696-9219 
territoriales más pequeñas como barrios o incluso intersecciones entre determinadas calles (por ejemplo, Weisburd et al., 2016).

El único trabajo realizado en España al respecto utiliza datos agregados a nivel de Área Básica Policial ${ }^{15}$ en Cataluña. En resumen, este trabajo señala que la distribución de las identificaciones no coincide de forma estricta con la distribución de la delincuencia (medida como delitos registrados o como victimización), como cabría esperar dado los objetivos legales que justifican el uso de la identificación policial (López-Riba, 2019).

Los datos disponibles en abierto tampoco señalan, por un lado, los motivos o indicios que llevaron al agente de policía a realizar la identificación, ni, por otro lado, que ocurrió después de la identificación (si se dejó marchar a la persona o se la detuvo, por ejemplo). Estos datos permitirían realizar análisis sobre el grado en que el uso de las identificaciones cumple con sus objetivos establecidos en la legislación: prevención de delitos y persecución de infracciones. Al respecto, se han llevado a cabo en España algunas investigaciones. Algunas se han centrado en la eficacia indagatoria, es decir hasta qué punto consiguen descubrir infracciones, señalando en general una eficacia baja o moderada (por ejemplo, García Añón et al., 2013; García España et al., 2016). También existe una investigación sobre su capacidad preventiva y esta muestra que las identificaciones pueden tener efectos disuasorios para una tipología delictiva de las estudiadas (delitos contra la propiedad) pero no para el resto (López-Riba, 2019).

Por último, tampoco hay fuentes oficiales que ofrezcan datos que intenten medir el impacto a medio y largo plazo de las identificaciones sobre la sociedad o sobre la propia policía. Aunque esto es difícil de medir, hay algunos ejemplos. La Enquesta Seguretat Pública de Catalunya pregunta, entre otras cosas, por la valoración que hacen los ciudadanos de los Mossos d'Esquadra y si la persona había tenido un contacto a iniciativa de la policía (como por ejemplo en una identificación, aunque no se pregunta exactamente por ello). Cruzando ambas preguntas se puede observar como la media de valoración de los Mossos

\footnotetext{
${ }^{15}$ Una ABP es una "unidad mínima, geográfica y poblacional, dotada de unos servicios básicos para la atención primaria de las demandas de prevención, seguridad ciudadana, control del tráfico e investigación de primer nivel." Ver: https://mossos.gencat.cat/es/els mossos desquadra/indicadors i qualitat/dades o bertes/AjudaDades-obertes-Mossos/
}

Revista Española de Investigación Criminológica

Artículo 1, Volumen 19 (2) (2021)

https://doi.org/10.46381/reic.v19i2.499

www.criminologia.net

ISSN: 1696-9219 
d'Esquadra es consistentemente menor en las diferentes ediciones de la encuesta entre las personas que tuvieron algún tipo de contacto involuntario ${ }^{16}$.

Las identificaciones pueden tener otro efecto a largo plazo importante más difícil de medir. Teniendo en cuenta que las identificaciones parecen afectar más a unas personas que a otras, esto puede provocar lo que Harcourt (2004) denomina ratchet effect: se identifica más a un determinado colectivo, lo que probablemente cause que se detenga más a personas que pertenecen a este colectivo, lo que lleva a que acaben en mayor proporción en los juzgados y que puede acabar con una sobrerrepresentación de personas de este colectivo en prisión. Problema también expuesto por Richardson et al. (2019).

\subsection{Detenciones}

Mientras que cualquier persona puede detener a otra en los supuestos del artículo 490 del Real Decreto de 14 de septiembre de 1882 por el que se aprueba la Ley de Enjuiciamiento Criminal (LECrim), los agentes de policía pueden hacerlo también en esas circunstancias y en otras establecidas en el artículo 492 de la LECrim, que además exige que para realizar una detención los agentes deben contar con "motivos racionalmente bastantes" de la existencia de una infracción penal y de la participación del sospechoso en esta.

En línea con su potencial lesividad (es uno de los dos únicos supuestos en los que legítimamente se puede privar de la libertad personal), este poder policial cuenta con una serie de garantías, más que ningún otro, que buscan someter a control legal estricto el ejercicio de esta potestad. Entre las garantías que se reconocen a la persona detenida están la prohibición de tortura o malos tratos (art. $15 \mathrm{CE}$ ), el límite de 72 horas, la obligación de informar a la persona detenida de sus derechos, el derecho a asistencia letrada, el procedimiento de habeas corpus (art. $17 \mathrm{CE}$ ), la tutela judicial (art. $24 \mathrm{CE}$ ) y otras garantías recogidas en la LECrim.

\footnotetext{
${ }^{16}$ Ver: https://interior.gencat.cat/ca/el departament/publicacions/seguretat/estudis-ienquestes/enquesta_de seguretat_publica_de catalunya/enquesta_de_seguretat_publica_de catalunya_2017/
}

Revista Española de Investigación Criminológica 
En comparación con en el caso de las identificaciones, los cuerpos de policía, sobre todo en el caso de policías estatales y autonómicas, ofrecen más datos sobre las detenciones que han practicado. Por ejemplo, en el Portal de Estadístico de Criminalidad del Ministerio del Interior (PECMI) se ofrecen datos anuales y trimestrales de personas detenidas e investigadas por todos los cuerpos de policía que operan en el territorio español (la misma información viene recogida en los AEMI y en los Balances Trimestrales de Seguridad). Esta información viene detallada según la tipología penal por la cual la persona ha sido detenida o investigada ${ }^{17}$, el sexo de la persona detenida, por grandes grupos de edad (o según si son adultos o menores), y por nacionalidad (si son españoles o extranjeros). A la hora de saber cuántas personas han sido detenidas efectivamente en España en un periodo concreto el propio portal señala algunas limitaciones referidas a la falta de datos para las detenciones referidas a algunas tipologías delictivas por parte de policías autonómicas ${ }^{18}$. Otra limitación al respecto es el hecho de presentar conjuntamente a las personas detenidas e investigadas.

Otros cuerpos de policía o las instituciones de las cuales dependen también publican datos sobre detenciones. Por ejemplo, en el Portal de Dades Obertes dels Mossos d'Esquadra se pueden encontrar datos de detenciones (no personas detenidas) mensuales, por Región Policial o Área Básica Policial y por tipología penal realizadas por los Mossos d’Esquadra. La web de Estadísticas Delictivas de la Ertzaintza ofrece datos sobre detenciones e investigaciones, de nuevo conjuntamente, anuales, por tipología penal, por territorio y por sexo de la persona detenida o investigada. En las MPFN se presentan también el número de detenciones e investigaciones, esta vez anualmente, por tipo de detención y por comisaria de los agentes que practicaron la detención. También es posible encontrar datos de detenciones de algunas policías locales ${ }^{19}$.

En suma, es imposible saber exactamente el número de personas detenidas o el número de detenciones en España con los datos que existen en abierto por las limitaciones

\footnotetext{
${ }^{17}$ Hasta 2010 se ofrecían datos sobre detenciones, a partir de entonces se ofrecen datos sobre detenidos e investigados, lo que además implica la imposibilidad de realizar comparaciones temporales o series históricas.

${ }^{18}$ Este problema de falta de información, y otras limitaciones, de los AEMI llevó a Marcelo Aebi y Antonia Linde a establecer la poca fiabilidad de los datos a la hora de cuantificar la delincuencia registrada por la policía en España (Aebi y Linde, 2010).

${ }^{19}$ Por ejemplo, en el Portal de Datos Abiertos del Ayuntamiento de Madrid.
}

Revista Española de Investigación Criminológica

Artículo 1, Volumen 19 (2) (2021)

https://doi.org/10.46381/reic.v19i2.499

www.criminologia.net

ISSN: 1696-9219 
apuntadas. Con los datos disponibles, del Portal de Estadístico de Criminalidad del Ministerio del Interior, únicamente se puede afirmar que en 2019 hubo como mínimo 411.903 detenciones y personas investigadas. La falta de datos en este caso es especialmente grave por ser una potestad que afecta a un derecho fundamental, como es la libertad, y que por lo tanto es necesaria una mayor transparencia en su ejercicio.

No existen trabajos empíricos que tengan por objetivo cuantificar las detenciones policiales practicadas en España, posiblemente o al menos en parte, debido a la falta de datos apuntada. Normalmente cuando se utilizan datos sobre detenciones se usan como una aproximación al fenómeno delictivo (por ejemplo, en Díez Ripollés, 2006), más que al propio ejercicio del poder policial.

Tampoco existen investigaciones encaminadas a comparar cuantitativamente el ejercicio de las detenciones en España con otros períodos u otros países (exceptuando el trabajo realizado en el caso de las detenciones en los European Sourcebook of Crime and Criminal Justice Statistics ${ }^{20}$ ). En la tabla 4 se presenta un ejercicio de comparación con Inglaterra y Gales y Estados Unidos para el año 2019.

Tabla 4

Comparación de la tasa de personas detenidas por cada 1000 habitantes

\begin{tabular}{cccc}
\hline País & $\begin{array}{c}\text { Tasa de personas } \\
\text { detenidas por cada 1000 } \\
\text { habitantes }\end{array}$ & $\begin{array}{c}\text { Fuente número } \\
\text { detenciones [año] }\end{array}$ & Fuente población [año] \\
\hline España & 8,74 & PECMI [2019] & $\begin{array}{c}\text { INE (2020) [segundo semestre } \\
\text { 2019] }\end{array}$ \\
\hline $\begin{array}{c}\text { Inglaterra y } \\
\text { Gales }\end{array}$ & 0,01 & $\begin{array}{c}\text { United Kingdom } \\
\text { Government (2020) } \\
{[2018 / 2019]}\end{array}$ & $\begin{array}{c}\text { Office for National Statistics } \\
(2020)[2019]\end{array}$ \\
\hline Estados & 24,41 & $\mathrm{FBI}^{21}[2019]$ & United States Census (2020) \\
Unidos & & & {$[2019]$} \\
\hline
\end{tabular}

\footnotetext{
${ }^{20} \mathrm{https}: / /$ wp.unil.ch/europeansourcebook/

${ }^{21} \mathrm{La}$ fuente se presenta en el próximo apartado.
} 
Si comparamos la tasa de personas detenidas por cada 1000 habitantes en España con la de Inglaterra y Gales vemos que la primera es 874 veces mayor. En cambio, si se toma como referencia Estados Unidos la tasa estadounidense es 2,79 veces mayor que la española ${ }^{22}$.

A diferencia de lo que ocurre con las identificaciones, los datos sobre personas detenidas, aunque limitados por los motivos anteriores, sí que suelen ofrecer características de estas como el sexo, la edad o la nacionalidad ${ }^{23}$. Pese a ello, no se ha analizado este aspecto en la literatura criminológica española. En la tabla 5 se muestran las tasas de personas detenidas por cada 1000 habitantes según las diferentes categorías demográficas.

\section{Tabla 5}

Tasa de personas detenidas por cada 1000 habitantes en función del sexo, edad y nacionalidad 2019

\begin{tabular}{cc}
\hline Grupo demográfico & Tasa de personas detenidas e investigadas \\
\hline Hombres & 13,48 \\
\hline Mujeres & 2,72 \\
\hline $14-17$ años & 10,42 \\
\hline $18-30$ años & 19,60 \\
\hline $31-40$ años & 15,79 \\
\hline $41-64$ años & 7,17 \\
\hline Más de 64 años & 1,16 \\
\hline Españoles & 7,23 \\
\hline Extranjeros & 21,44
\end{tabular}

Fuente: INE (2020) y PECMI

En la anterior tabla se puede ver como respecto al sexo la tasa es mayor para hombres que para mujeres (casi 5 veces). En cuanto a la edad, el grupo con una mayor tasa es el de 18 a 30 años, siendo esta tasa casi 2 veces mayor que para las personas de 14 a 17 años, 1,24 veces

\footnotetext{
${ }^{22}$ En esta comparación se debe tener en cuenta que en España se computan el número de detenciones y personas investigadas conjuntamente, que Inglaterra y Gales solo se cuenta el número de personas detenidas y en Estados Unidos se cuentan las detenciones.

${ }^{23}$ Es remarcable, en cuanto a la fiabilidad de los datos, que las sumas de las categorías de sexo o edad no se correspondan con el total de 411.903 detenciones y personas investigadas antes señalado.
}

Revista Española de Investigación Criminológica

Artículo 1, Volumen 19 (2) (2021)

https://doi.org/10.46381/reic.v19i2.499

www.criminologia.net

ISSN: 1696-9219 
mayor que para las personas de 31 a 40 años, 2,5 veces mayor que para las personas de 41 a 64 y casi 17 veces más alta que para las persones mayores de 64 años. En referencia a la nacionalidad, la tasa es casi 3 veces mayor para las personas extranjeras.

Relacionado con esto último, se deben tener en cuenta que a los extranjeros también pueden afectarle las detenciones relacionadas con la normativa de extranjería. Estas detenciones no se encuentran recogidas en las estadísticas del PECMI, allí solo se presentan las relacionadas con infracciones penales. En los IAMNPT sí que aparecen algunos datos sobre estas. Este tipo de detenciones son de especial interés en los estudios sobre la intersección entre el sistema penal y el control migratorio (por ejemplo, Brandariz-García \& Fernández-Bessa, 2017; García España, 2018; Martínez Escamilla \& Sánchez Tomás, 2019).

Los territorios para los que se ofrecen datos de detenciones suelen ser grandes unidades geográficas (provincias o CCAA) y como se ha señalado anteriormente las investigaciones criminológicas suelen interesarse por unidades geográficas más pequeñas, como los barrios. Puede ser por ello, en parte, por lo que no se cuenta con investigaciones sobre la distribución geográfica de las detenciones.

Respecto a la posibilidad de estudiar si se cumplen los fines (al menos, los oficiales) de la detención, los datos no permiten tratar estos aspectos de forma directa, porque no aparece el tipo de detención que se ha realizado (preventiva o procesal) ni se ofrece el número de personas detenidas a las que finalmente se ha condenado por delito. Tampoco existen investigaciones criminológicas al respecto.

Otro aspecto de interés respecto a las detenciones sería el impacto social a medio/largo plazo de la práctica que se esté llevando a cabo respecto a estas, más allá de los fines más o menos explícitos de este poder policial. Como en el caso de las identificaciones, las detenciones si se concentran más en determinados colectivos pueden acabar haciendo que esos colectivos sean, por un lado, estigmatizados, y, por otro, sobrerrepresentados en el sistema penal. También, dependiendo de cómo se lleven a cabo, podrían repercutir en una menor confianza en la policía. Sin embargo, no se encuentran disponibles datos sobre estos aspectos. Es por ello que en estos temas son más relevantes las investigaciones criminológicas al respecto, aunque no se han podido encontrar ejemplos en España.

Revista Española de Investigación Criminológica

Artículo 1, Volumen 19 (2) (2021)

https://doi.org/10.46381/reic.v19i2.499

www.criminologia.net

ISSN: 1696-9219 
Por último, en referencia a las detenciones, y por los motivos señalados anteriormente, también parecería importante contar con información oficial respecto hasta qué punto se respetan las garantías de las personas detenidas. En los IAMNPT puede encontrarse información al respecto.

\subsection{Otros poderes}

Pese a que este trabajo se ha centrado en aspectos detallados de las identificaciones y las detenciones, la policía tiene otras potestades y, en algunos, casos se cuenta con datos disponibles sobre su ejercicio, aunque nuevamente incompletos.

Por ejemplo, la policía tiene la capacidad de, cuando se perturbe la seguridad ciudadana, disolver reuniones o manifestaciones previo aviso ${ }^{24}$ (art. 23 de la LOPSC). Los datos más cercanos al ejercicio de esta potestad son aquellos que se presentan en los AEMI. Actualmente, en estos se indica el número de actuaciones de las Fuerzas y Cuerpos de Seguridad durante las manifestaciones comunicadas a o prohibidas por el Ministerio del Interior. Sin embargo, de nuevo, se plantea un problema a la hora de utilizar estos datos para efectivamente cuantificar (o comparar o realizar cualquier tipo de análisis) la facultad para disolver reuniones y manifestaciones. La información se presenta como número de actuaciones, sin especificar qué tipo de actuaciones ni a qué cuerpo policial se refieren. Blay (2013) muestra como en el pasado los AEMI recogían más información sobre la actuación de la policía en eventos de este tipo, por ejemplo, las detenciones o las personas heridas durante estas ${ }^{25}$.

Respecto al poder de denuncia/sanción de infracciones, la policía en España, -en otros contextos es diferente- no tiene la potestad para imponer sanciones ante infracciones penales o administrativas (por violaciones de la LOPSC, de la normativa de tráfico o de las ordenanzas municipales, por ejemplo). La policía denuncia y corresponde al órgano

\footnotetext{
${ }^{24}$ Excepto cuando la alteración a la seguridad ciudadana se produzca mediante el uso de armas, artefactos explosivos u objetos contundentes o peligrosos.

${ }^{25}$ Aunque la propia autora señala las limitaciones de estos datos y cómo la información va desapareciendo a lo largo del tiempo.
}

Revista Española de Investigación Criminológica

Artículo 1, Volumen 19 (2) (2021)

https://doi.org/10.46381/reic.v19i2.499

Www.criminologia.net

ISSN: 1696-9219 
competente imponer la pena o la sanción ${ }^{26}$. El PECMI recoge datos sobre infracciones a la LOPSC. Por ejemplo, se muestra como en 2019 hubo 244.313 propuestas de sanción que representaban una cuantía de aproximadamente 147 millones de euros.

Los pocos poderes policiales para los que tenemos datos cuantitativos, con muchas limitaciones, contrastan con el gran número de potestades policiales para las que no existe esta información: registros corporales, entrada y registro domiciliario, videovigilancia, registro de antecedentes policiales, aprehensión de objetos y sustancias, etc. Además, también se podría destacar la práctica nula información sobre el uso de la fuerza por parte de la policía en España.

\section{La situación en otros países}

Para ilustrar que algunos de los problemas señalados son salvables se van a exponer algunos ejemplos de fuentes de datos sobre poderes policiales disponibles en otros países y algunas de las investigaciones que permiten realizar esos datos.

\subsection{Las paradas y cacheos en Reino Unido}

El portal DATA.POLICE.UK (https://data.police.uk/data/) recoge datos sobre el ejercicio del poder de parar y cachear de todos los cuerpos policiales de Inglaterra, Gales e Irlanda del Norte. Estos datos incluyen información sobre: la fecha y el lugar exacto donde ocurrió la parada (con información sobre la latitud y longitud para mapearla), si se paró a una persona a pie o conduciendo un vehículo, las características de la persona parada (género, edad, etnia autodefinida y etnia percibida por el agente), si la parada formaba parte de una operación policial, bajo que legislación se justifica la parada, qué tipo de objeto se busca con el cacheo, el resultado de la parada y cacheo (nada, detención, sanción, etc.), etc. ${ }^{27}$

\footnotetext{
${ }^{26}$ Se podría considerar como excepción el procedimiento sancionador abreviado en materia de tráfico que permite en la práctica, vía consentimiento del afectado, que la policía sancione (artículo 94 del Real Decreto Legislativo 6/2015, de 30 de octubre, por el que se aprueba el texto refundido de la Ley sobre Tráfico, Circulación de Vehículos a Motor y Seguridad Vial).

27 Esta información, aunque menos detallada, la presenta el Home Office de forma anual en: https://www.gov.uk/government/collections/police-powers-and-procedures-england-and-wales
}

Revista Española de Investigación Criminológica

Artículo 1, Volumen 19 (2) (2021)

https://doi.org/10.46381/reic.v19i2.499

Www.criminologia.net

ISSN: 1696-9219 
Haciendo uso de esta fuente Ashby (2020) realiza un análisis en profundidad sobre las paradas y cacheos llevadas a cabo en Londres entre julio y septiembre de 2020. Entre otras cosas, este trabajo expone la evolución de las paradas y cacheos en Londres, mostrando una reducción con respecto a los meses anteriores; describe qué cuerpos policiales son los responsables del número de paradas y cacheos, siendo la Policía Metropolitana de Londres la principal protagonista (con un 97\%); también observa una reducción en el número de cacheos respecto al anterior cuatrimestre, expone los principales motivos que expresan los agentes de policía para realizarlos (el principal motivo es la búsqueda de drogas, con un 65,5\%), y advierte que el $76 \%$ de los cacheos acabaron sin ninguna acción policial consecuente; respecto a la distribución según las características personales, el estudio muestra como existe una desproporción importante en esta, siendo los hombres jóvenes negros los más afectados; y, finalmente, analiza como la mitad de las paradas y cacheos se concentran en solo el $9 \%$ de los barrios.

\subsection{Las detenciones en Estados Unidos}

El portal Crime Data Explorer del FBI (https://crime-data-explorer.app.cloud.gov/) contiene datos sobre detenciones a través del programa Uniform Crime Reporting (UCR), que es la fuente que obtiene información sistemática sobre la mayoría de cuerpos policiales de Estados Unidos. Los datos sobre detenciones se presentan de forma anual, por tipología delictiva, por sexo, por edad y por raza.

Snyder (2012) realiza un análisis exhaustivo de esos datos sobre detenciones entre 1990 y 2010 según la tipología delictiva, el sexo, la edad y la raza ${ }^{28}$. En cuanto a la tipología delictiva, en general se observa una tendencia creciente de la tasa de detenciones para la mayoría de tipologías delictivas en la década de los 90 y un descenso en la década de los 2000 (en algunos casos ese descenso se retrasa hasta los últimos años, como en el caso del consumo de drogas). En referencia al sexo, aunque las tendencias son similares, se muestra una mayor tasa de detenciones a hombres que a mujeres en todas las tipologías delictivas.

\footnotetext{
${ }^{28}$ Aunque en este último caso solo se presentan los números en absoluto no permitiendo la comparación.
}

Revista Española de Investigación Criminológica

Artículo 1, Volumen 19 (2) (2021)

https://doi.org/10.46381/reic.v19i2.499

www.criminologia.net

ISSN: 1696-9219 
Finalmente, respecto a la edad, los resultados son paralelos a la conocida curva edadparticipación delictiva (aumento en la adolescencia, pico en la mayoría de edad, descenso posterior) para todos los tipos delictivos.

Cabe señalar que los ejemplos son de Reino Unido y Estados Unidos, ya que en el resto de países la situación no es muy diferente a lo que ocurre en España (ver, para el caso de las paradas policiales, Open Society Justice Initiative, 2009). En cualquier caso, estos dos ejemplos muestran las posibilidades para la investigación criminológica de contar con fuentes de datos con información sistemática y detallada. También manifiestan la importancia de contar con este tipo de información para el cruce con otras fuentes y las oportunidades derivadas de esto.

\section{Limitaciones de los datos secundarios de la policía, transparencia y sus implicaciones}

Hasta ahora se han presentado algunas limitaciones de las fuentes de datos policiales que tienen que ver con la disponibilidad de estos. En muchas ocasiones no se trata de que los cuerpos policiales no recojan estos datos, pues se sabe que lo hacen de forma detallada y los utilizan como indicadores de su actuación (López Riba, 2019), sino de que no se publican. Sin embargo, trabajar con datos de la policía tiene otras limitaciones más generales. Estas se pueden diferenciar en limitaciones propias de los datos secundarios y limitaciones propias de los datos policiales. Por último, se tratará el problema de la transparencia.

El análisis de datos secundarios es un aspecto muy importante en la investigación criminológica, y en las ciencias sociales en general, principalmente por la dificultad y el coste de recoger datos primarios en las áreas que se estudian (Becker, 2017; Kleck et al., 2006). La principal limitación de utilizar datos secundarios es que estos suelen recogerse para otros motivos diferentes que el de realizar investigación. Por lo tanto, no se encuentran categorizados y organizados siguiendo los criterios de la disciplina que los utiliza para realizar análisis, sino los de la institución que las recoge (Becker, 2017; Kitsuse \& Cicourel, 1963). 
En el caso de los datos policiales esto es especialmente problemático. Están bien documentados los inconvenientes de utilizar datos sobre infracciones registradas por la policía o las detenciones como forma de medir el comportamiento delictivo. Desde problemas más generales, como las categorías utilizadas o la cifra oscura (Larrauri, 2018), pasando por problemas relacionados con los sesgos sobre qué infracciones y a quién persigue la policía, lo que lleva a considerar esa información como dirty data (Richardson et al., 2019), hasta problemas más concretos sobre transparencia y fiabilidad de los datos presentados (para el caso español, ver Aebi \& Linde, 2010; Serrano, 2019).

Sin embargo, frente a estos obstáculos se ha propuesto que las estadísticas policiales pueden ser utilizadas precisamente para estudiar el comportamiento de la policía (Becker, 2017; Kitsuse \& Cicourel, 1963). Por ejemplo, con los datos de delitos registrados por la policía difícilmente se puede medir la delincuencia general en un lugar y tiempo concreto, pero sí se puede extraer información sobre qué infracciones persigue más la policía. O si existe información sobre las características de las personas con las que interactúa la policía se pueden intentar estudiar los sesgos de la policía en su actividad rutinaria.

Ahora bien, aun cuando lo que se pretende es estudiar como fin en sí mismo el ejercicio de los poderes policiales, sigue habiendo inconvenientes al utilizar datos secundarios. Por un lado, no se puede asumir que estos datos reflejen de forma precisa la actividad policial rutinaria en la calle (Walker, 2001). Por ejemplo, en el caso de las paradas policiales están documentados casos de no correspondencia entre lo que la policía reporta y lo que realmente ocurre (Dixon, 1997; Spitzer, 1999, citado en Walker, 2001). Se debe tener en cuenta que los datos sobre el ejercicio de los poderes policiales se pueden recoger por motivos administrativos y políticos, es decir, para registrar el trabajo que se dice que se hace y para rendir cuentas a los superiores. Por lo tanto, existe un riesgo no desdeñable de manipulación (Becker, 2017). Por ejemplo, si se establece una política de reforma para intentar reducir el uso del perfil étnico, los agentes de policía pueden dejar de registrar algunas identificaciones problemáticas o mentir en la información que registran (poniendo que la persona es de nacionalidad española o que era blanca, cuando en realidad era extranjera o negra) (Walker, 2001).

Revista Española de Investigación Criminológica

Artículo 1, Volumen 19 (2) (2021)

https://doi.org/10.46381/reic.v19i2.499

www.criminologia.net

ISSN: 1696-9219 
Para finalizar, también se debe tratar el aspecto de la transparencia. De nada sirve recoger más datos si estos no se publican (o no se hacen accesibles bajo demanda a través de normativas de transparencia). Esto, en primer lugar, tiene importantes implicaciones democráticas. Las instituciones democráticas deben ser transparentes y deben rendir cuentas al público. En el caso de los poderes policiales esto es especialmente relevante, por los peligros inherentes al ejercicio de la violencia legítima del Estado y su inevitable tensión con derechos fundamentales. La falta de transparencia es un obstáculo al legítimo y necesario ejercicio de rendición de cuentas al que debe ser sometida la policía (Lennon \& Murray, 2018). En segundo lugar, y como se ha ido señalando a lo largo del artículo, la falta de una política de transparencia decidida dificulta la investigación criminológica al impedir el acceso a información que es muy difícil y costosa de obtener de otras formas.

Cabe señalar que se ha avanzado en los últimos años con respecto a la transparencia de los datos sobre la actividad del sistema penal. Algunos de los portales estadísticos que se han mencionado anteriormente, como el PECMI o el de los Mossos d'Esquadra, son un buen ejemplo. Además, a raíz de normativa estatal como la Ley 19/2013, de 9 de diciembre, de Transparencia, Acceso a la Información Pública y Buen Gobierno (también de normativas autonómicas o municipales), hoy es posible pedir datos a partir de los portales de transparencia de las diferentes administraciones ${ }^{29}$.

Aunque por los riesgos y limitaciones anteriormente señaladas a lo largo de este apartado y de todo el artículo, la transparencia no es el último paso. Es necesario también un ejercicio de auditoría y control de la actividad policial, y de su producción de datos, por parte de instituciones u organismos independientes como señalan Richardson et al. (2019) o como recomiendan organismos internacionales como la Oficina de las Naciones Unidas contra la Droga y el Delito (UNODC, 2011).

\footnotetext{
29 Algunos ejemplos: para la Administración General del Estado (https://transparencia.gob.es/transparencia/transparencia_Home/index.html), para la Generalitat valenciana (http://ue.gva.es/va/portal-de-transparencia) o para el Ayuntamiento de Madrid (https://transparencia.madrid.es/portal/site/transparencia).
} 


\section{Conclusiones}

En este trabajo se han pretendido exponer las principales fuentes de datos sobre poderes policiales y poner de manifiesto la falta de información y las carencias en los casos en los que está disponible. Para ejemplificar estos problemas se han elegido los casos de las identificaciones y las detenciones policiales. En referencia a las identificaciones, las limitaciones son muy pronunciadas. En primer lugar, desde el año 2015 no se cuentan con datos anuales de identificaciones llevadas a cabo por la Guardia Civil y el CNP (aunque de este último podemos encontrar alguna información en los IAMNPT). El problema no se acaba solo con los cuerpos policiales estatales puesto que también faltan datos de identificaciones de algunos cuerpos de policía autonómicos (Ertzaintza o Cuerpo General de Policía Canaria) y de la mayoría de policías locales (y teniendo en cuenta su peso relativo, esta carencia es importante). Incluso cuando hay datos disponibles estos suelen ser muy pobres en cuanto al nivel de detalle, no pudiendo conocer aspectos tan necesarios como algunas características de las personas identificadas (como su edad o su nacionalidad), las acciones (o falta de estas) que siguieron a la identificación, el lugar donde ocurrió la identificación (cuando se reporta el lugar suele corresponder a grandes unidades geográficas como CCAA o provincias), etc.

En cuanto a las detenciones, en este caso sí se cuenta con una fuente centralizada que reúne datos sobre detenciones y personas investigadas de la mayoría de cuerpos policiales que actúan en el territorio español, excepto para algunas tipologías delictivas por parte de los Mossos d'Esquadra ${ }^{30}$. Además, con las detenciones sí se presentan algunas características de las personas detenidas, como el sexo, la edad y la nacionalidad. Por lo demás, los datos sobre detenciones ofrecen los mismos problemas que los de identificaciones, a los que se añade la imposibilidad de separar las categorías de detenidos e investigados.

Posteriormente, se han presentado ejemplos de lo que ocurre en otros países, como contrapunto a las dificultades que se encuentran los investigadores en España. Concretamente se ha expuesto el caso de las paradas policiales en Reino Unido y las detenciones en Estados

\footnotetext{
${ }^{30}$ Lo que impide conocer, con los datos disponibles en abierto, el número de detenciones policiales en un periodo concreto en España.
}

Revista Española de Investigación Criminológica

Artículo 1, Volumen 19 (2) (2021)

https://doi.org/10.46381/reic.v19i2.499

Www.criminologia.net

ISSN: 1696-9219 
Unidos, ofreciendo fuentes de datos y ejemplos de investigaciones que se han realizado a partir de estas. Cabe señalar, como se ha apuntado anteriormente, que estas dificultades en el acceso a datos de la actividad policial son comunes en la mayoría de países. Los ejemplos expuestos de Reino Unido y Estados Unidos son excepciones en el contexto internacional. Estas diferencias entre países seguramente se deban a la mayor tradición de rendición de cuentas de la actividad policial en el contexto anglosajón (ver, por ejemplo, Open Society Justice Initiative, 2009).

Por último, se ha advertido sobre las limitaciones añadidas de trabajar con datos secundarios producidos por la policía. Las principales limitaciones al respecto es que estos datos se recogen con fines diferentes al de permitir la investigación criminológica y los posibles sesgos derivados de ello. Una vez advertidos los problemas de los datos secundarios se ha señalado la necesidad de adoptar una política de transparencia más decidida, pese a los avances en ello, porque esto, por un lado, podría dar un impulso a la investigación criminológica española, pero principalmente porque es un deber democrático someter el ejercicio de los poderes policiales a escrutinio público. A esto último faltaría añadir las recomendaciones, por ejemplo de la UNODC (2011), que se refieren a la auditoría externa de la producción de estos datos.

\section{Referencias}

Aebi, M. F. \& Linde, A. (2010). El misterioso caso de la desaparición de las estadísticas policiales españolas. Revista Electrónica de Ciencia Penal y Criminología, 12, 1-30.

APDHA \& IPAZ-UGR. (2016). Identificaciones basadas en perfil étnico en Granada: estudio realizado mediante observación y entrevistas directas. Disponible en https://www.apdha.org/identificaciones-policiales-basadas-en-perfil-etnico-engranada/

Ashby, M. (2020). Stop and search in London - July to September 2020. Disponible en https://discovery.ucl.ac.uk/id/eprint/10115766/1/2020-Q3.pdf

Becker, H. (2017). Evidence. The University of Chicago Press.

Blay, E. (2011). El control policial de las protestas en España. InDret: Revista para el Análisis del Derecho, 4, 1-32.

Bradford, B. (2014). Policing and social identity: Procedural justice, inclusion and cooperation between police and public. Policing and Society, 24(1), 22-43. https://doi.org/10.1080/10439463.2012.724068

Revista Española de Investigación Criminológica

Artículo 1, Volumen 19 (2) (2021)

https://doi.org/10.46381/reic.v19i2.499

www.criminologia.net

ISSN: 1696-9219 
Brandariz-García, J. A. \& Fernández-Bessa, C. (2017). The managerial turn: The transformation of Spanish migration control policies since the onset of the economic crisis. The Howard Journal of Crime and Justice, 56(2), 198-219.

Brodeur, J. P. (2010). The policing web. Oxford University Press.

de la Serna, C. (2014). Uso de perfiles étnicos por parte de la policía en España: Una oportunidad histórica de erradicarlos. Debates Jurídicos, 3, 1-12.

Díez-Ripollés, J. L. (2006). Algunos rasgos de la delincuencia en España a comienzos del siglo XXI. Revista Española de Investigación Criminológica, 4, 1-19. https://doi.org/10.46381/reic.v4i0.28

Dixon, D. (1997). Law in policing: Legal regulation and police practices. Clarendon Press.

Duce, M. \& Lillo, R. (2020). Controles de identidad realizados por Carabineros: Una aproximación empírica y evaluativa sobre su uso en Chile. Revista de Estudios de la Justicia, 33, 167-203.

Fernández Molina, E. \& Bartolomé, R. (2020). Juvenile crime drop: What is happening with youth in Spain and why? European Journal of Criminology, 17(3), 306-331. https://doi.org/10.1177/1477370818792383

García Añón, J. G., Bradford, B., García, J. A., Gascón, A. \& Llorente, A. (2013). Identificación policial por perfil étnico en España: Informe sobre experiencias y actitudes en relación con las actuaciones policiales. Tirant lo Blanch.

García España, E. (2018). Enfoque criminológico de las migraciones. Editorial Síntesis.

García España, E., Arenas, L. \& Miller, J. (2016). Identificaciones policiales y discriminación racial en España: Evaluación de un programa para su reducción. Tirant lo Blanch.

Guillén, F. (2012). Policia i seguretat. Servei de Publicacions Universitat Autònoma de Barcelona.

Harcourt, B. E. (2004). Rethinking racial profiling: A critique of the economics, civil liberties, and constitutional literature, and of criminal profiling more generally. The University of Chicago Law Review, 71(4), 1275-1381.

INE (2020). Cifras de población. Disponible en https://www.ine.es/dyngs/INEbase/es/operacion.htm?c=Estadistica_C\&cid=125473 6176951\&menu $=$ ultiDatos\&idp $=1254735572981$

Kitsuse, J. I. \& Cicourel, A. V. (1963). A note on the uses of official statistics. Social Problems, 11, 131-139.

Kleck, G., Tark, J. \& Bellows, J. J. (2006). What methods are most frequently used in research in criminology and criminal justice? Journal of Criminal Justice, 34, 147152. https://doi.org/10.1016/j.jcrimjus.2006.01.007

Larrauri, E. (2018). Introducción a la criminología y al sistema penal (2a edición). Editorial Trotta.

Lennon, G. \& Murray, K. (2018). Under-regulated and unaccountable? Explaining variation in stop and search rates in Scotland, England and Wales. Policing and Society, 28(2), 157-174. https://doi.org/10.1080/10439463.2016.1163359

Revista Española de Investigación Criminológica

Artículo 1, Volumen 19 (2) (2021)

https://doi.org/10.46381/reic.v19i2.499

Www.criminologia.net

ISSN: 1696-9219 
López-Riba, J. M. (2019). Las identificaciones policiales en España: Un análisis crítico desde la criminología. [Tesis doctoral, Universidad Pompeu Fabra]. Disponible en https://www.tesisenred.net/handle/10803/666512\#page=1

López-Riba, J. M. (2021). Distributive justice and police legitimacy: The demographic and geographical distribution of police ID checks in Spain. Política Criminal, 16(31), 145-161.

Martínez Escamilla, M. \& Sánchez Tomás, J. M. (2019). Los requerimientos de identificación racistas y detenciones en el control migratorio. Revista Crítica Penal y Poder, 18, 1-10.

Office for National Statistics (2020). Population estimates. Disponible en https://www.ons.gov.uk/peoplepopulationandcommunity/populationandmigration/p opulationestimates

Open Society Justice Initiative. (2009). Addressing ethnic profiling by police: A report on the Strategies for Effective Police Stop and Search Project. Disponible en https://www.opensocietyfoundations.org/sites/default/files/profiling_20090511.pdf

Richardson, R., Schultz, J. M. \& Crawford, K. (2019). Dirty data, bad predictions: How civil rights violations impact police data, predictive policing systems, and justice. New York University Law Review Online, 94, 15-55.

Ríos, J., Segovia, J. L. \& Etxebarria, X. (2015). Manual para la defensa de los derechos y las libertades ante la intervención de la policía. Universidad Pontificia Comillas.

Serrano, A. (2019). Dudosa fiabilidad de las estadísticas policiales sobre criminalidad en España. Revista de Derecho Penal y Criminología, 6, 425-454.

Snyder, H. N. (2012). Arrest in the United States, 1990-2010. Disponible en https://www.bjs.gov/content/pub/pdf/aus9010.pdf

United Kingdom Government (2020). Policing statistics. Disponible en https://www.gov.uk/government/collections/policing-statistics

UNODC (2011). Handbook on police accountability, oversight and integrity. Disponible en https://www.unodc.org/pdf/criminal_justice/Handbook_on_police_Accountability_ Oversight_and_Integrity.pdf

United States Census (2020). United States 2019 population estimates. Disponible en https://www.census.gov/

Walker, S. (2001). Searching for the denominator: Problems with police traffic stop data and an early warning system solution. Justice Research and Policy, 3(1), 63-95. https://doi.org/10.3818/JRP.3.1.2001.63

Weisburd, D., Wooditch, A., Weisburd, S. \& Yang, S.-M. (2016). Do stop, question, and frisk practices deter crime? Evidence at microunits of space and time. Criminology \& Public Policy, 15(1), 31-56. https://doi.org/10.1111/1745-9133.12172

\section{Revista Española de Investigación Criminológica}

Artículo 1, Volumen 19 (2) (2021)

https://doi.org/10.46381/reic.v19i2.499

www.criminologia.net

ISSN: 1696-9219 


\section{Agradecimientos}

Me gustaría agradecer, en primer lugar, a mis compañeros y compañeras del grupo de investigación de la Universidad de Girona así como a Úrsula Ruiz Cabello, sus correcciones y comentarios a las primeras versiones de este artículo. En segundo lugar, también me gustaría agradecer a los/as revisores/as anónimos/as las sugerentes observaciones y propuestas de cambio durante el proceso de revisión. Por último, agradezco especialmente a todo el equipo editorial de la REIC por su trabajo en la gestión y publicación de este artículo.

\section{Financiamiento}

Para la realización de este trabajo no se ha recibido ningún tipo de financiación.

José María López-Riba es doctor en Derecho/Criminología por la Universidad Pompeu Fabra, donde realizó su tesis doctoral sobre las identificaciones policiales. Actualmente es investigador postdoctoral en el Departamento de Derecho público de la Universidad de Girona. Además, imparte clases de diferentes materias en el Grado en Criminología de la misma universidad. Sus líneas de investigación incluyen, entre otras, las actuaciones policiales, el derecho a la educación reglada en los centros penitenciarios, la discrecionalidad judicial y la selectividad del sistema penal.

https://orcid.org/0000-0003-0520-710X 


\section{Anexo}

\section{Tabla A}

Fuentes de datos con información sobre el ejercicio de los poderes policiales en España

Fuentes internacionales

\section{DATAUNODC}

\begin{tabular}{|c|c|c|c|c|}
\hline $\begin{array}{l}\text { Institución } \\
\text { responsable }\end{array}$ & Datos disponibles & Detalle & Formatos & Enlace \\
\hline \multirow[t]{3}{*}{ UNODC } & $\begin{array}{l}\text { Personas que han tenido } \\
\text { contacto formal con la policía } \\
\text { o justicia (sospechosos, } \\
\text { detenidos y amonestados) }\end{array}$ & recuento y tasas; por país o continente; anualmente & \multirow[t]{3}{*}{ xlsx } & \multirow[t]{3}{*}{ https://dataunodc.un.org/ } \\
\hline & Droga incautada & por país o continente; anualmente; por tipo de droga & & \\
\hline & Armas ilegales incautadas & $\begin{array}{l}\text { por país o continente; anualmente; por tipo de arma; } \\
\text { por tipo de justificación legal }\end{array}$ & & \\
\hline \multicolumn{5}{|l|}{ EUROSTAT } \\
\hline $\begin{array}{l}\text { Institución } \\
\text { responsable }\end{array}$ & Datos disponibles & Detalle & Formatos & Enlace \\
\hline $\begin{array}{l}\text { European } \\
\text { Comission }\end{array}$ & $\begin{array}{l}\text { Personas que han tenido } \\
\text { contacto formal con la policía } \\
\text { o justicia (sospechosos, } \\
\text { detenidos y amonestados) }\end{array}$ & $\begin{array}{l}\text { recuento y tasas; por país; anualmente; por sexo; por } \\
\text { grupo de edad (adultos vs jóvenes); nacionalidad } \\
\text { (nacionales vs. extranjeros) }\end{array}$ & $\begin{array}{l}\text { xls, csv, } \\
\text { html, pc } \\
\text { axis, sav, } \\
\text { tsv, pdf }\end{array}$ & https://ec.europa.eu/eurostat/data/database \\
\hline
\end{tabular}

Fuentes nacionales

\section{PORTAL ESTADÍSTICO DE CRIMINALIDAD}

\begin{tabular}{|c|c|c|c|c|}
\hline $\begin{array}{l}\text { Institución } \\
\text { responsable }\end{array}$ & Datos disponibles & Detalle & Formatos & Enlace \\
\hline $\begin{array}{l}\text { Ministerio del } \\
\text { Interior }\end{array}$ & Detenidos e investigados & $\begin{array}{l}\text { por CCAA o provincias; anualmente o } \\
\text { trimestralmente; por tipología penal; por sexo; por } \\
\text { grupos de edad o adultos vs menores; nacionalidad } \\
\text { (nacionales vs. extranjeros) }\end{array}$ & $\begin{array}{l}\text { pc axis, } \\
\text { xls/xlsx, } \\
\text { csv }\end{array}$ & $\begin{array}{l}\text { https://estadisticasdecriminalidad.ses.mir.es } \\
\text { /publico/portalestadistico/ }\end{array}$ \\
\hline
\end{tabular}

Revista Española de Investigación Criminológica

Artículo 1, Volumen 19 (2) (2021)

https://doi.org/10.46381/reic.v19i2.499

www.criminologia.net

ISSN: 1696-9219 


\begin{tabular}{|c|c|c|c|c|}
\hline & $\begin{array}{l}\text { Propuestas de sanción y } \\
\text { cuantía LOPSC }\end{array}$ & por CCAA o provincias; anualmente; por infracción & & \\
\hline \multicolumn{5}{|c|}{ ANUARIOS ESTADÍSTICOS DEL MINISTERIO DEL INTERIOR } \\
\hline $\begin{array}{l}\text { Institución } \\
\text { responsable }\end{array}$ & Datos disponibles* & Detalle & Formatos & Enlace \\
\hline \multirow{7}{*}{$\begin{array}{l}\text { Ministerio del } \\
\text { Interior }\end{array}$} & Detenidos e investigados & $\begin{array}{l}\text { por CCAA o provincias; anualmente o } \\
\text { trimestralmente; por tipología penal; por sexo; por } \\
\text { grupos de edad o adultos vs menores; nacionalidad } \\
\text { (nacionales vs. extranjeros) }\end{array}$ & \multirow{7}{*}{ pdf } & \multirow{7}{*}{$\begin{array}{l}\text { http://www.interior.gob.es/web/archivos-y- } \\
\text { documentacion/documentacion-y- } \\
\text { publicaciones/anuarios-y-estadisticas }\end{array}$} \\
\hline & $\begin{array}{l}\text { Propuestas de sanción y } \\
\text { cuantía LOPSC }\end{array}$ & por CCAA o provincias; anualmente; por infracción & & \\
\hline & Identificaciones & $\begin{array}{l}\text { por CCAA o provincias; anualmente; según lugar de } \\
\text { la identificación }\end{array}$ & & \\
\hline & $\begin{array}{l}\text { Intervenciones en } \\
\text { manifestaciones }\end{array}$ & $\begin{array}{l}\text { anualmente; por tipo de manifestación (comunicada } \\
\text { o prohibida) }\end{array}$ & & \\
\hline & Incautaciones de droga & anualmente; por tipo de droga & & \\
\hline & $\begin{array}{l}\text { Intervenciones en materia de } \\
\text { armas y explosivos }\end{array}$ & anualmente; por tipo de arma & & \\
\hline & $\begin{array}{l}\text { Actuaciones en materia de } \\
\text { seguridad vial }\end{array}$ & $\begin{array}{l}\text { por CCAA o provincias; anualmente; por tipo de } \\
\text { actuación }\end{array}$ & & \\
\hline \multicolumn{5}{|c|}{ INFORMES ANUALES DEL MECANISMO NACIONAL DE PREVENCIÓN DE LA TORTURA } \\
\hline $\begin{array}{l}\text { Institución } \\
\text { responsable }\end{array}$ & Datos disponibles* & Detalle & Formatos & Enlace \\
\hline \multirow{4}{*}{$\begin{array}{l}\text { Defensor del } \\
\text { Pueblo }\end{array}$} & Detenciones policiales & $\begin{array}{l}\text { por CCAA o provincias; anualmente; por cuerpo } \\
\text { policial; por grupos de edad (adultos vs. menores); } \\
\text { por sexo; por nacionalidad (nacionales vs. } \\
\text { extranjeros); según régimen de incomunicación }\end{array}$ & \multirow{4}{*}{ pdf } & \multirow{4}{*}{$\begin{array}{l}\text { https://www.defensordelpueblo.es/informes } \\
\text { /resultados-busqueda- } \\
\text { informes/?tipo_documento=informe_mnp }\end{array}$} \\
\hline & $\begin{array}{l}\text { Identificaciones practicadas } \\
\text { por el CNP }\end{array}$ & por CCAA o provincias; anualmente & & \\
\hline & $\begin{array}{l}\text { Detenciones Ley de } \\
\text { Extranjería }\end{array}$ & por CCAA o provincias; anualmente & & \\
\hline & Denegaciones de entrada & $\begin{array}{l}\text { por puesto fronterizo de salida; por país de } \\
\text { procedencia; anualmente }\end{array}$ & & \\
\hline
\end{tabular}

Revista Española de Investigación Criminológica

Artículo 1, Volumen 19 (2) (2021)

https://doi.org/10.46381/reic.v19i2.499

www.criminologia.net

ISSN: 1696-9219 


\begin{tabular}{|c|c|c|c|c|}
\hline & $\begin{array}{l}\text { Muertes, intentos de suicidio } \\
\text { o autolisis durante detención } \\
\text { policial } \\
\text { Denuncias interpuestas por } \\
\text { malos tratos/tortura por parte } \\
\text { de agentes de policía }\end{array}$ & $\begin{array}{l}\text { por cuerpo policial; anualmente } \\
\text { anualmente; por cuerpo policial (CNP o Guardia } \\
\text { Civil); por derecho afectado }\end{array}$ & & \\
\hline \multicolumn{5}{|c|}{ Fuentes autonómicas } \\
\hline \multicolumn{5}{|c|}{ PORTAL DADES OBERTES MOSSOS D'ESQUADRA } \\
\hline $\begin{array}{l}\text { Institución } \\
\text { responsable }\end{array}$ & Datos disponibles & Detalle & Formatos & Enlace \\
\hline \multirow{6}{*}{$\begin{array}{l}\text { Mossos } \\
\text { d'Esquadra }\end{array}$} & Detenciones & mensualmente; por ABP o RP; por tipología penal & \multirow{6}{*}{$\begin{array}{l}\text { xls, csv, } \\
\text { json }\end{array}$} & \multirow{6}{*}{$\begin{array}{l}\text { https://mossos.gencat.cat/ca/els_mossos_de } \\
\text { squadra/indicadors_i qualitat/dades_oberte } \\
\text { s/cataleg_dades_obertes/ }\end{array}$} \\
\hline & Media de patrullas diarias & mensualmente; por ABP o RP & & \\
\hline & Incidentes atendidos & mensualmente; por ABP o RP; por ámbito & & \\
\hline & $\begin{array}{l}\text { Tiempo medio de respuesta a } \\
\text { incidentes graves }\end{array}$ & mensualmente; por RP & & \\
\hline & Controles de velocidad & mensualmente; por ABP o RP & & \\
\hline & $\begin{array}{l}\text { Atestados por accidentes de } \\
\text { tráfico }\end{array}$ & mensualmente; por ABP o RP & & \\
\hline \multicolumn{5}{|c|}{ ESTADÍSTICA POLICIAL } \\
\hline $\begin{array}{l}\text { Institución } \\
\text { responsable }\end{array}$ & Datos disponibles & Detalle & Formatos & Enlace \\
\hline \multirow{8}{*}{$\begin{array}{l}\text { Departament } \\
\text { d'Interior }\end{array}$} & Detenciones & anualmente; por tipología penal & \multirow{8}{*}{ pdf } & \multirow{8}{*}{$\begin{array}{l}\text { https://mossos.gencat.cat/ca/els_mossos_de } \\
\underline{\text { squadra/indicadors_i_qualitat/estadistica/ }}\end{array}$} \\
\hline & Personas investigadas & anualmente & & \\
\hline & Personas denunciadas & anualmente & & \\
\hline & Identificaciones & anualmente & & \\
\hline & Controles de paso & anualmente & & \\
\hline & Atestados instruidos & anualmente & & \\
\hline & Incidentes atendidos & anualmente; por ámbito & & \\
\hline & $\begin{array}{l}\text { Horas destinadas a } \\
\text { prevención }\end{array}$ & anualmente; por ámbito & & \\
\hline
\end{tabular}

Revista Española de Investigación Criminológica

Artículo 1, Volumen 19 (2) (2021)

https://doi.org/10.46381/reic.v19i2.499

www.criminologia.net

ISSN: 1696-9219 


\begin{tabular}{|c|c|c|c|c|}
\hline & Protecciones de víctimas & anualmente & & \\
\hline \multicolumn{5}{|c|}{ ESTADÍSTICAS DELICTIVAS } \\
\hline $\begin{array}{l}\text { Institución } \\
\text { responsable }\end{array}$ & Datos disponibles & Detalle & Formatos & Enlace \\
\hline Ertzaintza & Detenciones e investigaciones & $\begin{array}{l}\text { anualmente; por tipología penal; por territorio; por } \\
\text { sexo }\end{array}$ & xlsx & $\begin{array}{l}\text { https://www.ertzaintza.eus/lfr/web/ertzaint } \\
\underline{\text { za/estadisticas-delictivas }}\end{array}$ \\
\hline \multicolumn{5}{|c|}{ MEMORIA POLICIA FORAL DE NAVARRA } \\
\hline $\begin{array}{l}\text { Institución } \\
\text { responsable }\end{array}$ & Datos disponibles & Detalle & Formatos & Enlace \\
\hline \multirow{8}{*}{$\begin{array}{l}\text { Policía Foral de } \\
\text { Navarra }\end{array}$} & Detenciones e investigaciones & anualmente; por tipo de detención; por comisaria; & \multirow{8}{*}{ pdf } & \multirow{8}{*}{$\begin{array}{l}\text { https://www.poderjudicial.es/cgpj/es/Tema } \\
\text { s/Estadistica-Judicial/Estadistica-por- } \\
\text { temas/Actividad-de-otros-tribunales- } \\
\text { nacionales-y-de-otros- } \\
\text { agentes/Seguridad/Memoria-Policia-Foral- } \\
\text { de-Navarra/ }\end{array}$} \\
\hline & Identificaciones & anualmente; por tipo (personas o vehículos) & & \\
\hline & Incautaciones de drogas & anualmente; por tipo de droga & & \\
\hline & Denuncias & anualmente; por reglamento; por comisaria & & \\
\hline & $\begin{array}{l}\text { Protección de } \\
\text { manifestaciones }\end{array}$ & anualmente & & \\
\hline & Pruebas seguridad vial & anualmente; por tipo (alcoholemia o drogas) & & \\
\hline & $\begin{array}{l}\text { Actuaciones protección } \\
\text { medioambiente }\end{array}$ & anualmente; por tipo de actuación & & \\
\hline & Actuaciones juego y ocio & anualmente; por tipo de actuación & & \\
\hline \multicolumn{5}{|c|}{ Fuentes municipales** } \\
\hline \multicolumn{5}{|c|}{ PORTAL DE DATOS ABIERTOS DEL AYUNTAMIENTO DE MADRID } \\
\hline $\begin{array}{l}\text { Institución } \\
\text { responsable }\end{array}$ & Datos disponibles & Detalle & Formatos & Enlace \\
\hline \multirow{3}{*}{$\begin{array}{l}\text { Ayuntamiento } \\
\text { de Madrid }\end{array}$} & Detenidos e investigados & anualmente; por distrito; por tipología penal & \multirow{3}{*}{ xlsx } & \multirow{3}{*}{$\begin{array}{l}\text { https://datos.madrid.es/portal/site/egob/me } \\
\text { nuitem.c05c1f754a33a9fbe4b2e4b284f1a5 } \\
\text { a0/?vgnextoid=bffff1d2a9fdb410VgnVCM } \\
\text { 2000000c205a0aRCRD\&vgnextchannel=3 } \\
\underline{\text { 74512b9ace9f310VgnVCM100000171f5a0 }} \\
\underline{\text { aRCRD }}\end{array}$} \\
\hline & Propuestas de sanción & $\begin{array}{l}\text { anualmente; por distrito; por propiedad } \\
\text { industrial/intelectual, "infracción alimentaria", } \\
\text { consumo de alcohol en la vía pública, locales, }\end{array}$ & & \\
\hline & Promedio de patrullas & $\begin{array}{l}\text { por día de la semana; por horario (mañana, tarde y } \\
\text { noche) }\end{array}$ & & \\
\hline \multicolumn{5}{|c|}{ ANUARIOS ESTADÍSTICOS DE LA CIUDAD DE BARCELONA } \\
\hline
\end{tabular}

Revista Española de Investigación Criminológica

Artículo 1, Volumen 19 (2) (2021)

https://doi.org/10.46381/reic.v19i2.499

www.criminologia.net

ISSN: 1696-9219 


\begin{tabular}{|c|c|c|c|c|}
\hline $\begin{array}{l}\text { Institución } \\
\text { responsable }\end{array}$ & Datos disponibles & Detalle & Formatos & Enlace \\
\hline \multirow{5}{*}{$\begin{array}{l}\text { Ayuntamiento } \\
\text { de Barcelona }\end{array}$} & $\begin{array}{l}\text { Denuncias policía de } \\
\text { circulación }\end{array}$ & anualmente; por distrito; según reglamento & & \multirow{5}{*}{$\begin{array}{l}\text { https://www.bcn.cat/estadistica/castella/dad } \\
\text { es/anuaris/index.htm }\end{array}$} \\
\hline & $\begin{array}{l}\text { Denuncias policía } \\
\text { administrativa }\end{array}$ & anualmente; por distrito; según reglamento & & \\
\hline & Pruebas de alcoholemia & anualmente; por distrito; por motivo & & \\
\hline & Drogo-test & anualmente; por distrito; por motivo & & \\
\hline & $\begin{array}{l}\text { Actuaciones de ayuda y } \\
\text { asistencia }\end{array}$ & anualmente; por distrito; por motivo & & \\
\hline
\end{tabular}

* Dependen del documento concreto. Se exponen ejemplos de algunos de ellos.

** Se exponen dos ejemplos, los de las dos ciudades más grandes de España que cuentan con los mayores cuerpos de policía local.

Revista Española de Investigación Criminológica

Artículo 1, Volumen 19 (2) (2021)

https://doi.org/10.46381/reic.v19i2.499

www.criminologia.net

ISSN: 1696-9219 